Supporting Information for

\title{
Polyaniline Hybrid Nanofibers via Green Interfacial Polymerization for All-Solid-State Symmetric Supercapacitors
}

\author{
Gayatri Konwara , Saurav Ch. Sarma ${ }^{b, c}$, Debajyoti Mahanta ${ }^{a^{*}}$, Sebastian C. Peter ${ }^{b, c^{*}}$ \\ aDepartment of Chemistry, Gauhati University, Guwahati, 781014, Assam, India

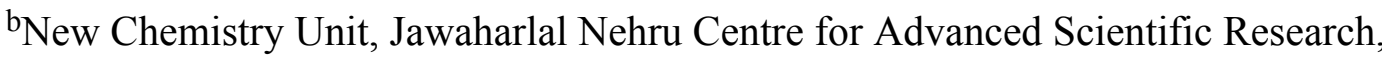 \\ Bangalore, India
}

'School of Advanced Materials, Jawaharlal Nehru Centre for Advanced Scientific Research,

Bangalore 560 064, India

*Corresponding author. Phone: 080-22082998, Fax: 080-22082627

sebastiancp@jncasr.ac.in (S. C. Peter)

Synthesis of PANI by chemical oxidation- The monomer aniline ( $5 \mathrm{~g}, 4.90 \mathrm{~mL}$ ) was dissolved in $1 \mathrm{M} \mathrm{HCl}(150 \mathrm{~mL})$ and stirred in an ice bath for half an hour. The oxidizing agent APS (12.25 g) was dissolved in $1 \mathrm{M} \mathrm{HCl}(50 \mathrm{~mL})$ and kept in an ice bath for 15 minutes. After that the APS solution was taken in a burette and added to the aniline solution drop wise under constant stirring at temperature $0-5{ }^{\circ} \mathrm{C}$. The reaction mixture was stirred for 6 hours in a magnetic stirrer after complete addition of APS in the aniline solution. The green product obtained was washed with distilled water for several times and finally with acetone to remove low molecular weight oligomers followed by drying in air and then kept in a desiccator. Thus we get PANI in the ES form.

Table S1. Composition of various elements in PANI-SO obtained from the EDAX measurement.

\begin{tabular}{ccccccccc}
\hline Element & Weight \% & Atomic \% & Net Int. & Error \% & Kratio & Z & A & F \\
& & & & & & & & \\
\hline C K & 58.51 & 70.59 & 1169.16 & 9.97 & 0.1267 & 1.0446 & 0.2073 & 1.0000 \\
N K & 10.91 & 11.28 & 106.09 & 17.65 & 0.0106 & 1.0173 & 0.0959 & 1.0000 \\
O K & 10.69 & 9.69 & 234.89 & 13.53 & 0.0170 & 0.9938 & 0.1597 & 1.0000 \\
S K & 7.10 & 3.21 & 635.61 & 3.04 & 0.0631 & 0.8726 & 1.0000 & 1.0193 \\
ClK & 12.79 & 5.23 & 937.02 & 2.88 & 0.1044 & 0.8290 & 0.9798 & 1.0046 \\
\hline
\end{tabular}




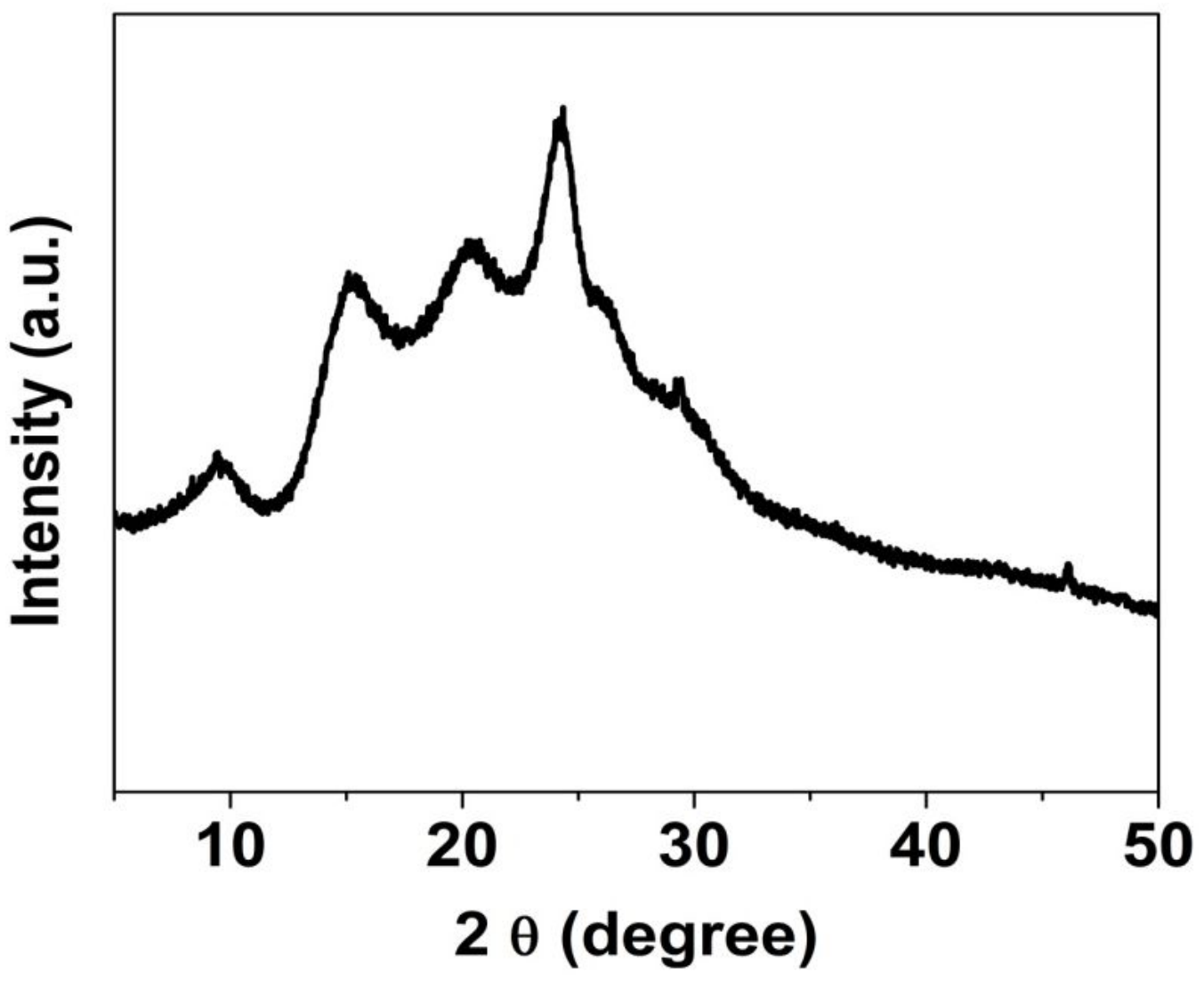

Figure S1. PXRD pattern of PANI (ES).

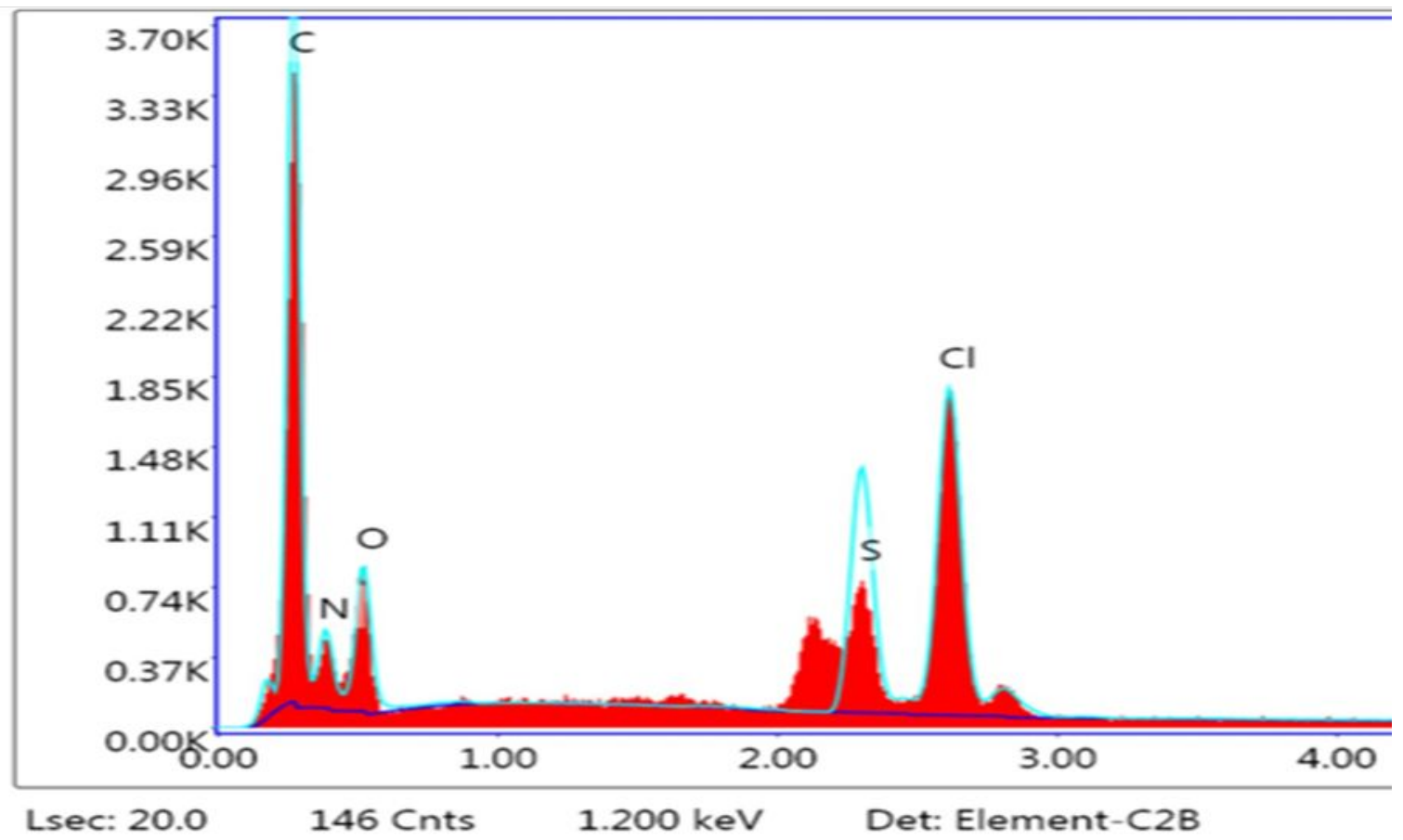

Figure S2. EDAX spectrum of PANI-SO. 

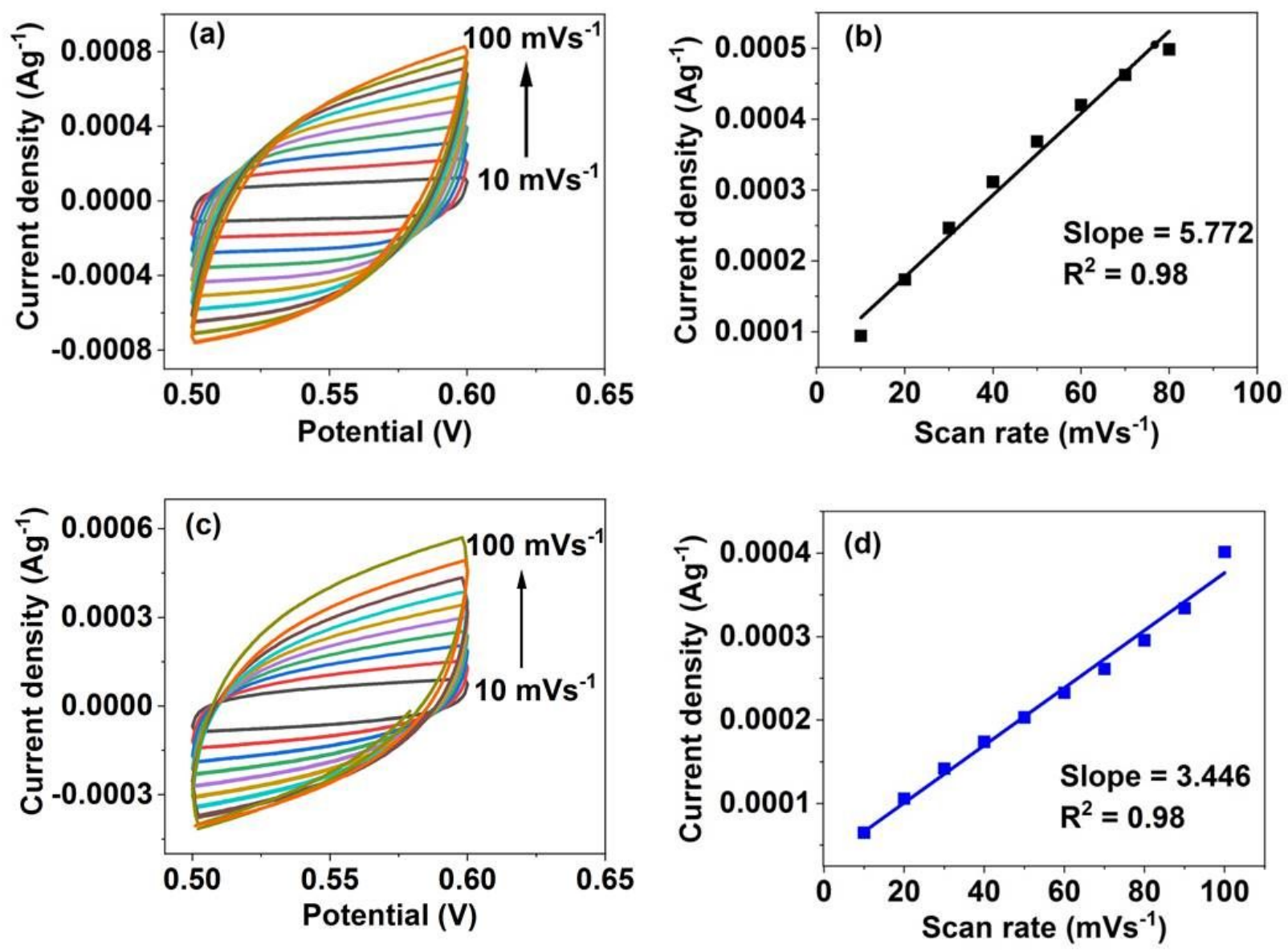

Figure S3. (a) and (c) CV s of PANI-SO and PANI-CHCl $\mathrm{PH}_{3}$ at various scan rates from 10 to $100 \mathrm{mVs}^{-1}$ within the potential range of $0.50-0.6 \mathrm{~V}$ respectively. (b) and (d) Linear fitting of current density vs. scan rates at a potential of $0.55 \mathrm{~V}$ for PANI-SO and PANI-CHCl $\mathrm{P}_{3}$ respectively.

\section{Comparison of PANI-SO with other reported PANI nanofibers}

A comparison study of specific capacitance of PANI-SO with other PANI nanofibers was also carried out. This comparison is shown in the table S2 for three electrode system. It is seen that PANI nanofibers synthesized by electrochemical oxidation gives the higher specific capacitance value than that of conventional chemical methods. Wang et.al have synthesized PANI nanowire by electrochemical polymerization method. ${ }^{1}$ The specific capacitance obtained was $950 \mathrm{~F} / \mathrm{g}$ at current density of $1 \mathrm{~A} / \mathrm{g}$. They have used $1 \mathrm{M} \mathrm{HClO}_{4}$ as an electrolyte for three electrode system. Hollow PANI nanofibers were synthesized by Miao et.al. ${ }^{2}$ Hollow PANI nanofibers show the specific capacitance of $601 \mathrm{~F} / \mathrm{g}$ at $1 \mathrm{~A} / \mathrm{g}$ current density. Both of 
these values of specific capacitance are higher than that of PANI-SO. PANI based CNT were reported as an electrode material by Yang et.al. ${ }^{3}$ They have used $30 \mathrm{wt} . \% \mathrm{KOH}$ as an electrolyte. PANI based CNT gives the specific capacitance of $163 \mathrm{~F} / \mathrm{g}$ at current density of $0.1 \mathrm{~A} / \mathrm{g}$. PANI-SO shows the better specific capacitance at $1 \mathrm{~A} / \mathrm{g}$ than that of PANI based CNT electrode material. Zhang et. al have also reported PANI nanofibers as supercapacitive material. They have carried out the charge/discharge analysis in $1 \mathrm{M}$ tetraethylammonium tetrafluoroborate/propylene carbonate solution. PANI nanofibers give the specific capacitance of $160 \mathrm{~F} / \mathrm{g}$ at lower current density of $0.4 \mathrm{~A} / \mathrm{g}$. Simotwo et.al have also synthesized PANI nanofibers which gives the specific capacitance of $308 \mathrm{~F} / \mathrm{g}$ at $0.5 \mathrm{~A} / \mathrm{g} .^{5}$ Similarly, Guan et. al have synthesized PANI nanofibers by interfacial polymerization method. Specific capacitance of $548 \mathrm{~F} / \mathrm{g}$ was obtained at $0.18 \mathrm{~A} / \mathrm{g} .{ }^{6}$ By comparing all these reported values of specific capacitance we can say that PANI-SO which was synthesized by modified interfacial polymerization method is a promising supercapactive material.

Table S2. Comparison study of specific capacitance of PANI-SO with other PANI nanofibers

\begin{tabular}{|l|l|l|l|l|l|}
\hline Material & Method & Electrolyte & $\begin{array}{l}\text { Current } \\
\text { density } \\
(\mathbf{A} / \mathbf{g})\end{array}$ & $\begin{array}{l}\text { Specific } \\
\text { capacitance } \\
\text { (F/g) }\end{array}$ & Ref. \\
\hline $\begin{array}{l}\text { PANI } \\
\text { nanowire }\end{array}$ & Electrochemical & $1 \mathrm{M} \mathrm{HClO}_{4}$ & 1 & 950 & 1 \\
\hline $\begin{array}{l}\text { Hollow } \\
\text { PANI } \\
\text { nanofibers }\end{array}$ & Chemical & $1 \mathrm{M} \mathrm{H}_{2} \mathrm{SO}_{4}$ & 1 & 601 & 2 \\
\hline $\begin{array}{l}\text { PANI base } \\
\text { CNT }\end{array}$ & Chemical & 30 wt.\% KOH & 0.1 & 163 & 3 \\
\hline $\begin{array}{l}\text { PANI } \\
\text { nanofibers }\end{array}$ & Chemical & $\begin{array}{l}1 \mathrm{M} \\
\text { tetraethylammoniu } \\
\text { m } \\
\text { tetrafluoroborate/pr } \\
\text { opylene carbonate } \\
\text { solution }\end{array}$ & 0.4 & 160 & 4 \\
\hline $\begin{array}{l}1 \mathrm{M} \mathrm{H}_{2} \mathrm{SO}_{4} \\
\text { nanofibers }\end{array}$ & Chemical & 0.5 & 308 & 5 \\
\hline $\begin{array}{l}\text { PANI } \\
\text { nanofiber }\end{array}$ & Chemical & $1 \mathrm{M} \mathrm{H}_{2} \mathrm{SO}_{4}$ & 0.18 & 548 & 6 \\
\hline
\end{tabular}

References 
(1). Kai Wang, K.; J Huang, J.; Wei, Z. Conducting Polyaniline Nanowire Arrays for High Performance Supercapacitors. J. Phys. Chem. C. 2010, 114, 8062-8067.

(2). Miao, Y-E.; Fan, W.; Chen, D.; Liu, T. High-Performance Supercapacitors Based on Hollow Polyaniline Nanofibers by Electrospinning. ACS Appl. Mater. Interfaces. 2013, 5, $4423-4428$.

(3). Yang, M.; Cheng, B.; Song, H.; Chen, X. Preparation and electrochemical performance of polyaniline-based carbon nanotubes as electrode material for supercapacitor. Electrochim. Acta. 2010, 55, 7021-7027.

(4). Zhang, H.; Zhao, Q.; Zhou, S.; Liu, N.; Wang, X.; Li, J.; Wang, F. Aqueous dispersed conducting polyaniline nanofibers: Promising high specific capacity electrode materials for supercapacitor. J. Power. Sources. 2011, 196, 10484- 10489.

(5). Simotwo, S.K.; DelRe, C.; Kalra, V. Supercapacitor Electrodes Based on High-Purity Electrospun Polyaniline and Polyaniline-Carbon Nanotube Nanofibers. ACS Appl. Mater. Interfaces. 2016, 8, 21261-2126.

(6). Guana, H.; Fan, L-Z.; Zhang, H.; Qu, X. Polyaniline nanofibers obtained by interfacial polymerization for high-rate supercapacitors. Electrochim. Acta. 2010, 56, 964-968. 